This item was submitted to Loughborough's Research Repository by the author.

Items in Figshare are protected by copyright, with all rights reserved, unless otherwise indicated.

\title{
Kalman-gain aided particle PHD filter for multi-target tracking
}

PLEASE CITE THE PUBLISHED VERSION

http://dx.doi.org/10.1109/TAES.2017.2690530

PUBLISHER

IEEE

VERSION

VoR (Version of Record)

\section{PUBLISHER STATEMENT}

This work is made available according to the conditions of the Creative Commons Attribution 3.0 Unported (CC BY 3.0) licence. Full details of this licence are available at: http://creativecommons.org/licenses/by/3.0/

\section{LICENCE}

CC BY 3.0

\section{REPOSITORY RECORD}

Daniyan, Abdullahi, Yu Gong, Sangarapillai Lambotharan, Pengming Feng, and Jonathon Chambers. 2019. "Kalman-gain Aided Particle PHD Filter for Multi-target Tracking". figshare. https://hdl.handle.net/2134/24704. 


\section{Kalman-Gain Aided Particle PHD Filter for Multitarget Tracking}

\author{
ABDULLAHI DANIYAN ${ }^{\mathbb{D}}$, Student Member, IEEE \\ YU GONG, Member, IEEE \\ SANGARAPILLAI LAMBOTHARAN, Senior Member, IEEE \\ Loughborough University, Loughborough, U.K.
}

PENGMING FENG, Member, IEEE

JONATHON CHAMBERS, Fellow, IEEE

Newcastle University, Newcastle upon Tyne, U.K.

We propose an efficient sequential Monte Carlo probability hypothesis density (PHD) filter which employs the Kalman-gain approach during weight update to correct predicted particle states by minimizing the mean square error between the estimated measurement and the actual measurement received at a given time in order to arrive at a more accurate posterior. This technique identifies and selects those particles belonging to a particular target from a given PHD for state correction during weight computation. Besides the improved tracking accuracy, fewer particles are required in the proposed approach. Simulation results confirm the improved tracking performance when evaluated with different measures.

Manuscript received November 11, 2015; revised September 19, 2016 and December 18, 2016; released for publication March 24, 2017. Date of publication April 5, 2017; date of current version October 10, 2017.

DOI. No. 10.1109/TAES.2017.2690530

Refereeing of this contribution was handled by B.-N. Vo.

This work was supported in part by the Engineering and Physical Sciences Research Council under Grant EP/K014307/1, in part by the MOD University Defence Research Collaboration in Signal Processing, U.K., and in part by the Petroleum Technology Development Fund, Nigeria.

Authors' addresses: A. Daniyan, Y. Gong, and S. Lambotharan are with the School of Electronic, Electrical and Systems Engineering, Loughborough University, Loughborough LE11 3TU, U.K., E-mail: (a.daniyan@lboro.ac.uk; y.gong@lboro.ac.uk; s.lambotharan@lboro.ac. uk); P. Feng and J. Chambers are with the School of Electrical and Electronic Engineering, Newcastle University, Newcastle upon Tyne NE1 7RU,U.K.,E-mail: (p.feng2@newcastle.ac.uk; jonathon.chambers@ newcastle.ac.uk). (Corresponding author: Abdullahi Daniyan.)

0018-9251 (C) 2017 CCBY

\section{INTRODUCTION}

Multitarget tracking (MTT) is essential in many application areas, such as motion-based recognition, automated security, navigation and surveillance, medical imaging, traffic control, and human-computer interaction [1]-[3]. MTT belongs to a class of dynamic state estimation problems [3]-[5]. In MTT, targets can appear and disappear randomly in time and this results in a varying and unknown number of targets and their corresponding states. Furthermore, not all measurements received by sensors at each time instance are due to existing targets. The sensor may pick up detections as false alarms due to clutter or may even miss some detections. As a result, the measurements received at each time step are corrupted and consist of indistinguishable measurements that may be either target originated or due to clutter. Therefore, the main objective of MTT is to be able to jointly estimate target states and number of targets from a set of corrupted observations.

Furthermore, because there is no particular ordering between measurements received and target states at each time step in terms of association, both the received measurements and target states can be represented as finite sets [6]-[9]. The modeling of target states and observations as a random finite set (RFS) allows for the use of the Bayesian filtering approach (as an optimal multitarget filter) to estimate the multitarget states in the presence of clutter, missed detections, and association uncertainty [6]-[9]. Tractable alternatives to the optimal multitarget filters include the RFS-based probability hypothesis density (PHD) filter, the cardinalized PHD (CPHD) filter [8]-[10], the multitarget multi-Bernoulli (MeMBer) filter, and its cardinality-balanced version, the CBMeMBer filter [8], [9], [11]. Both the CPHD in [10] and the CBMeMBer in [11] have been shown to have better performance than the MeMBer filter in [8]. The CBMeMBer filter was proposed specifically to address the pronounced bias in the cardinality estimate of the MeMBer filter. For more details on other tractable RFS-based MTT methods, the reader is referred to [12]-[15].

The PHD filter is a recursion that propagates the posterior intensity of the RFS of targets in time [6]. The integral of the PHD is the expected number of targets in a measurable region, and the peaks of the PHD function provide the estimates of the target states [6], [8], [9]. The PHD filter is able to track time-varying multiple targets without the need to explicitly associate measurements to tracks. In the literature, the PHD filter has been implemented in two distinct fashions, that is, as the Gaussian mixture PHD (GM-PHD) filter [16] and the sequential Monte Carlo PHD (SMC-PHD) filter [17]. In the GM-PHD filter implementation, the PHD is assumed to be a GM while in the SMC-PHD filter implementation, the PHD is approximated by a set of weighted particles and does not need any further

\footnotetext{
${ }^{1}$ The MeMBer filter is a recursion that propagates (approximately) the multitarget posterior density and is based on the assumption that every multitarget posterior is a MeMBer process [8], [11].
} 
assumptions. The SMC-PHD filter is therefore more suitable for tracking in nonlinear and non-Gaussian environments.

In SMC filter design, the choice of importance density function from which samples are drawn to avoid sample degeneracy and impoverishment is of crucial importance [18]. Furthermore, in MTT which involves multiple modalities, if particles are in clusters representing the modes of the posterior, the iterative process of randomly drawing samples from proposal distributions results in random fluctuations in the total weight attributed to each mode [19]. In addition, the errors associated with the estimation of the weights of each mode will increase in magnitude with time [19]. These errors arise due to the stochastic nature of drawing samples from the proposal distribution and the stochasticity of the resampling process [19]. These two processes greatly influence the performance of SMC filters. SMC filters are further affected by how well the state space of targets is populated with samples. In addition, Vo et al. [20] argued that the mean squared error (MSE) of the SMC-PHD filter is inversely proportional to the number of samples. In [18], it is shown that the optimal importance density function is the posterior. In many cases it is difficult to sample from the optimal importance density. As an attempt to solve the importance sampling problem, Maskell and Julier [19] proposed an optimized proposal distribution for SMC filters with multiple modes in general. However, this approach tends to be problem specific. In [21], Yoon et al. proposed the Gaussian mixture unscented sequential Monte Carlo probability hypothesis density (GM-USMC-PHD) filter which uses the GM representation to approximate the importance sampling function and the predictive density functions via the unscented information filter (UIF). Additionally, in [22] and [23] the auxiliary SMC-PHD filter and its improved version, the auxiliary particle PHD (AP-PHD) filter are proposed, respectively. Both try to use the auxiliary particle approach to incorporate the measurement into the importance sampling function. This, however, involves double computation on the measurement and more samples are required to populate the state space in order to make the importance sampling function more viable.

However, it is also possible to construct suboptimal approximations to the optimal importance density by using local linearization techniques [18]. As a realization of this, the unscented Kalman particle PHD filter was proposed in [24] for the joint tracking of multitargets. It tries to use the unscented Kalman filter (UKF) in the prediction step. This allowed for the inclusion of the latest measurement to draw particles. Similarly, Ma et al. [25] proposed the Kalman particle PHD filter for multitarget visual tracking, which uses the Kalman filter to construct the proposal density also in the prediction step. Furthermore, Tang et al. [26] presented an improvement to the SMC-PHD filter, which incorporates the latest measurements into the resampling step by using the UKF.

Additionally, there are other works in the literature that combine the implementation of the GM and particle PHD filter into one filter such as the GM particle PHD filter in
[27] and [29] and the Gaussian mixture SMC-PHD in [30]. These methods attempt to combine the advantages of both GM-PHD and SMC-PHD filters. The methods give some level of performance improvement without easing computational burden or the number of particles. Also, it may be possible to implement the Markov Chain Monte Carlo (MC) sampling method in the update stage of the SMCPHD filter as a way of asymptotically approximating the posterior. However, this approach will require even more particles, as these extra particles will be used to perform some sort of random walk in order to achieve maximum $a$ posteriori estimate of target states but no guarantees exist about it yielding good point estimates [31]. Recently, Zheng et al. [32] proposed a data-driven SMC-PHD filter for MTT. The method tries to segment the measurements available at each time step into measurements due to persistent targets and measurements due to newborn targets. Again this does not help to reduce the number of particles but rather, more particles are required to populate regions of interest.

It is desirable, therefore, to have an efficient filter that can provide for particle state correction for any proposal distribution using fewer particles. This gives the motivation for the Kalman-gain aided sequential Monte Carlo probability hypothesis density (KG-SMC-PHD) filter. The KGSMC-PHD filter provides for the particle state correction of the predicted multitarget state. This is achieved with the application of the Kalman state update technique on selected particles to minimize the MSE between the estimated measurements and actual measurement.

In this paper, we propose an SMC-PHD filter with a validation threshold to select promising particles and to guide them to regions of high likelihood using the Kalman gain, irrespective of the importance density function. This method seeks to minimize the MSE between the estimated measurements due to selected particles and the actual measurements to achieve a more efficient SMC-PHD filter with less computational complexity. This allows fewer particles to be used to populate the state space and at the same time achieve improved tracking performance as opposed to the standard SMC-PHD filter.

The remainder of this paper is organized as follows. In Section II, the MTT problem is presented in terms of process and measurement models. Section III presents the idea of the importance density function and highlights some common choices of proposal distributions. In Section IV, the PHD filter recursion is presented and explained followed by a description of the standard SMC-PHD filter implementation. Next, Section V presents our proposed KG-SMCPHD filter. Simulation results together with discussions are presented in Section VI. Finally, conclusions are drawn in Section VII.

\section{MTT PROBLEM FORMULATION}

The MTT problem relates to that of modeling a dynamical system. Two models are generally used, the state evolution model and the measurement model. 


\section{A. State Model}

A nonlinear system governed by the state evolution model is considered

$$
\mathbf{x}_{k}=\mathbf{f}_{k-1}\left(\mathbf{x}_{k-1}, \mathbf{v}_{k}\right)
$$

where $\mathbf{x}_{k}$ denotes the $t$ th target state at discrete time $k, \mathbf{v}_{k}$ is an independent and identically distributed (i.i.d.) process noise vector, and $\mathbf{f}_{k-1}(\cdot)$ is the nonlinear system transition function. Then, the multitarget state at time $k$ can be written as

$$
\boldsymbol{X}_{k}=\left\{\mathbf{x}_{1, k}, \ldots, \mathbf{x}_{T, k}\right\} \in E_{s}
$$

where $T$ is the number of targets present at each time $k$, and $E_{s}$ denotes the state space.

\section{B. Measurement Model}

Let the multitarget cumulative measurement sequence up to time $K$ be $\boldsymbol{Z}_{1: K}: \mathbf{Z}_{1}, \mathbf{Z}_{2}, \ldots, \mathbf{Z}_{K} \subset E_{o}$. Measurements consist of both target-originated measurements and false alarms due to clutter. Then, the multitarget measurement set at time $k$ in the observation space is

$$
\boldsymbol{Z}_{k}=\left\{\mathbf{z}_{1, k}, \ldots, \mathbf{z}_{\alpha, k}\right\} \bigcup\left\{\mathbf{c}_{1, k}, \ldots \mathbf{c}_{\beta, k}\right\} \subset E_{o}
$$

where $\left\{\mathbf{z}_{1, k}, \ldots, \mathbf{z}_{\alpha, k}\right\}$ denotes the target-originated measurement set with number of measurements $\alpha$; $\left\{\mathbf{c}_{1, k}, \ldots \mathbf{c}_{\beta, k}\right\}$ denotes the false measurement set with the number of measurements $\beta$, and $E_{o}$ denotes the observation space. The $t$ th target-originated nonlinear measurement model is given as

$$
\mathbf{z}_{k}=\mathbf{h}_{k}\left(\mathbf{x}_{k}, \mathbf{n}_{k}\right)
$$

where $\mathbf{h}_{k}(\cdot)$ is a nonlinear function and $\mathbf{n}_{k}$ is an i.i.d. process noise vector.

\section{IMPORTANCE DENSITY FUNCTION}

In this section, we focus on proposal distributions and their role in SMC methods in general.

\section{A. Importance Sampling}

MC methods for numerical integration deal with problems of the form

$$
g=\int_{\Re^{n}} f(\mathbf{y}) \pi(\mathbf{y}) d \mathbf{y}
$$

where $\pi(\mathbf{y})$ is such that $\pi(\mathbf{y}) \geq 0$ and integrates to unity

$$
\int_{\Re^{n}} \pi(\mathbf{y}) d \mathbf{y}=1
$$

is a pdf.

It is also the assumption that it is possible to generate $N \gg 1$ samples distributed according to the probability density $\pi(\mathbf{y})$. The MC estimate of the integral (5) is formed by taking the average over the set of samples

$$
\hat{g}=\frac{1}{N} \sum_{i=1}^{N} f\left(\mathbf{y}_{i}\right)
$$

where $N$ is assumed to be large. However, $\pi(\mathbf{y})$ is not usually a familiar density and so it is difficult to generate samples directly from it. When the latter is the case, the integral of (5) can be solved by letting $q(\mathbf{y})$ be a proposal distribution or importance density which is easy to generate samples and with the assumption that $\pi(\mathbf{y})>0 \Rightarrow q(\mathbf{y})>0$ for all $\mathbf{y} \in \mathfrak{R}^{n}$. Under this assumption, (5) becomes

$$
g=\int_{\Re^{n}} f(\mathbf{y}) \pi(\mathbf{y}) d \mathbf{y}=\int_{\Re^{n}} f(\mathbf{y}) \frac{\pi(\mathbf{y})}{q(\mathbf{y})} q(\mathbf{y}) d \mathbf{y} .
$$

An MC estimate is then computed by generating $N \gg 1$ samples from $q(\mathbf{y})$ instead of $\pi(\mathbf{y})$ and forming a weighted sum

$$
\hat{g}=\frac{1}{N} \sum_{i=1}^{N} f\left(\mathbf{y}_{i}\right) w\left(\mathbf{y}_{i}\right)
$$

where $w\left(\mathbf{y}_{i}\right) \propto \frac{\pi\left(\mathbf{y}_{i}\right)}{q\left(\mathbf{y}_{i}\right)}$ are the associated weights [33]. To sum up, given a distribution $\pi(\mathbf{y})$ that is difficult to sample from, importance sampling facilitates sampling from $\pi(\mathbf{y})$ by sampling from an alternate distribution $q(\mathbf{y})$ known as proposal distribution but weighting appropriately.

\section{B. Importance Densities}

Some common choices of importance density in SMC methods are given below.

1) Transitional Prior (TP): This is the most popular choice of suboptimal proposal distribution for SMC-PHD filters and particle filters in general because its implementation is easy and straightforward [34]. This choice requires sampling from the dynamic prior, i.e.,

$$
q\left(\mathbf{x}_{k} \mid \mathbf{x}_{k-1}^{l}, \mathbf{z}_{k}\right)=p\left(\mathbf{x}_{k} \mid \mathbf{x}_{k-1}^{l}\right) .
$$

2) Extended Particle Filter (EPF): Given that the measurement model of (4) is nonlinear, but Gaussian, it is possible to use a proposal distribution that exploits a linear approximation to the posterior [19] in the same way as the extended Kalman filter (EKF) uses a local linearization about its estimates. The proposal distribution is then given as

$$
q\left(\mathbf{x}_{k} \mid \mathbf{x}_{k-1}^{l}, \mathbf{z}_{k}\right)=\mathcal{N}\left(\mathbf{x}_{k} ; \mathbf{u}_{k}, \mathbf{A}_{k}\right)
$$

where

$$
\begin{aligned}
\mathbf{u}_{k} & =\mathbf{f}_{k-1}\left(\mathbf{x}_{k-1}\right)+\mathbf{A}_{k} \mathbf{H}_{k}^{T} \mathbf{R}_{k}^{-1}\left(\mathbf{z}_{k}-\mathbf{h}\left(\mathbf{f}_{k-1}\left(\mathbf{x}_{k-1}\right)\right)\right) \\
\mathbf{H}_{k} & =\left.\frac{\partial \mathbf{h}}{\partial \mathbf{x}_{k}}\right|_{\mathbf{f}_{k-1}\left(\mathbf{x}_{k-1}\right)}
\end{aligned}
$$

where $\mathbf{A}_{k}$ and $\mathbf{R}_{k}$ denote state and measurement covariances, respectively, and $\mathbf{H}_{k}$ is the measurement transformation matrix.

3) Unscented Particle Filter (UPF): As an alternative to the EPF, an unscented transform can be used to calculate the mean $\mathbf{h}\left(\mathbf{f}_{k-1}\left(\mathbf{x}_{k-1}\right)\right)$ and covariance $\mathbf{H}_{k}$ by generating sigma points and applying a transform such that the new generated samples have $\mathbf{f}_{k-1}\left(\mathbf{x}_{k-1}\right)$ as mean and $\mathbf{P}_{k-1}$ as covariance. $\mathbf{h}\left(\mathbf{f}_{k-1}\left(\mathbf{x}_{k-1}\right)\right)$ is then evaluated at each sigma point and $\mathbf{H}_{k}$ computed from these samples [19]. 


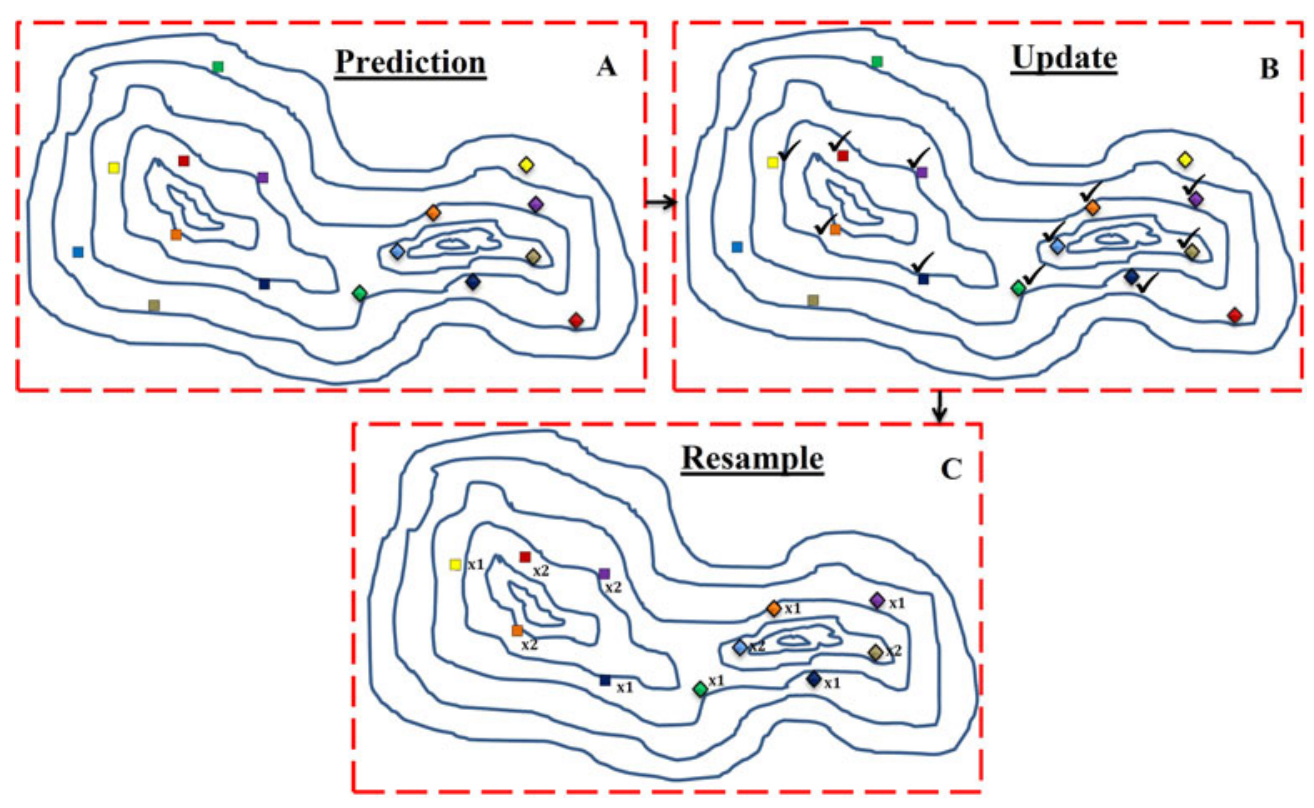

Fig. 1. Schematic representation of the standard SMC-PHD filter showing the 2-D state space of the PHD of two targets populated with particles. The contours represent the state space of targets. The contour centers and number of centers represent the mode and cardinality of targets, respectively. Boxes A, B, and $\mathbf{C}$ represent various stages of the filter. The square-shaped and diamond-shaped particles are for target 1 and target 2 , respectively. The colours stand for different particle states. The particles marked with " $\sqrt{ }$ " in $\mathbf{B}$ denote particles with higher weight for when the latest observation arrives.

\section{PROBABILITY HYPOTHESIS DENSITY}

\section{A. PHD Filter}

The PHD $D_{\Lambda}$ of a given RFS $\Lambda$ is the first-order moment of $\Lambda$ and is given by [6], [8], [9] the following equation:

$$
D_{\Lambda}(\mathbf{x})=\mathbf{E}\left\{\delta_{\Lambda}(\mathbf{x})\right\}=\int \delta_{\mathbf{X}}(\mathbf{x}) P_{\Lambda}(d \mathbf{X})
$$

where $\mathbf{E}\{\cdot\}$ is the statistical expectation operator and $\delta_{\Lambda}(\mathbf{x})=\sum_{\mathbf{y} \in \Lambda} \delta_{\mathbf{y}(\mathbf{x})}$ is the random density representation of $\Lambda . P_{\Lambda}$ is the probability measure of the RFS. The PHD filter is a recursion of the PHD, $D_{k \mid k}$ that is associated with the multitarget posterior density $p\left(\mathbf{X}_{k} \mid \mathbf{Z}_{k}\right)$, and

$$
p\left(\boldsymbol{X}_{k} \mid \boldsymbol{Z}_{k}\right) \propto p\left(\boldsymbol{Z}_{k} \mid \boldsymbol{X}_{k}\right) p\left(\boldsymbol{X}_{k} \mid \boldsymbol{Z}_{k-1}\right)
$$

where $p\left(\boldsymbol{Z}_{k} \mid \boldsymbol{X}_{k}\right)$ and $p\left(\boldsymbol{X}_{k} \mid \boldsymbol{Z}_{k-1}\right)$ denote the multitarget likelihood and prior density, respectively.

The prediction formula of the PHD, $D_{k \mid k}$ is given as [8], [9] follows:

$$
\begin{aligned}
& D_{k \mid k-1}\left(\mathbf{x}_{k} \mid \boldsymbol{Z}_{k-1}\right)=\gamma_{k}\left(\mathbf{x}_{k}\right) \\
& \quad+\int \phi_{k \mid k-1}\left(\mathbf{x}_{k}, \mathbf{x}_{k-1}\right) D_{k-1 \mid k-1}\left(\mathbf{x}_{k-1} \mid \boldsymbol{Z}_{k-1}\right) d \mathbf{x}_{k-1}
\end{aligned}
$$

with the factor

$$
\begin{aligned}
\phi_{k \mid k-1}\left(\mathbf{x}_{k}, \mathbf{x}_{k-1}\right)= & p_{S}\left(\mathbf{x}_{k-1}\right) f_{k \mid k-1}\left(\mathbf{x}_{k}, \mathbf{x}_{k-1}\right) \\
& +b_{k \mid k-1}\left(\mathbf{x}_{k}, \mathbf{x}_{k-1}\right)
\end{aligned}
$$

where $\gamma_{k}(\cdot)$ is the PHD of the spontaneous birth, $p_{S}(\cdot)$ is the probability of the target survival, $f_{k \mid k-1}\left(\mathbf{x}_{k}, \mathbf{x}_{k-1}\right)$ is the single target motion model, and $b_{k \mid k-1}\left(\mathbf{x}_{k}, \mathbf{x}_{k-1}\right)$ is the PHD of the spawned targets.
The update formula is given as

$$
\begin{aligned}
& D_{k \mid k}\left(\mathbf{x}_{k} \mid \boldsymbol{Z}_{k}\right) \\
& =\left[v\left(\mathbf{x}_{k}\right)+\sum_{\mathbf{z} \in Z_{k}} \frac{\psi_{k, \mathbf{z}}\left(\mathbf{x}_{k}\right)}{\kappa_{k}(\mathbf{z})+\left\langle D_{k \mid k-1}, \psi_{k, \mathbf{z}}\right\rangle}\right] D_{k \mid k-1}\left(\mathbf{x}_{k} \mid \boldsymbol{Z}_{k-1}\right)
\end{aligned}
$$

with $v\left(\mathbf{x}_{k}\right)=1-p_{D}\left(\mathbf{x}_{k}\right), \psi_{k, \mathbf{z}}\left(\mathbf{x}_{k}\right)=p_{D}\left(\mathbf{x}_{k}\right) g\left(\mathbf{z} \mid \mathbf{x}_{k}\right)$, and $\kappa_{k}(\mathbf{z})=\lambda_{k} c_{k}(\mathbf{z})$; where $p_{D}\left(\mathbf{x}_{k}\right)$ and $v\left(\mathbf{x}_{k}\right)$ denote the probability of target detection and nondetection for a given $\left(\mathbf{x}_{k}\right)$, respectively, $g\left(\mathbf{z} \mid \mathbf{x}_{k}\right)$ is the measurement likelihood function for the single target, $\kappa_{k}(\mathbf{z})$ is the clutter intensity, $\lambda_{k}$ is the average number of Poisson clutter points per scan, and $c_{k}(\mathbf{z})$ is the probability density over the state-space of the clutter point; $\langle\cdot, \cdot\rangle$ denotes inner product and is computed as [8], [9]

$$
\left\langle D_{k \mid k-1}, \psi_{k, \mathbf{z}}\right\rangle=\int D_{k \mid k-1}\left(\mathbf{x}_{k} \mid \boldsymbol{Z}_{k-1}\right) \psi_{k, \mathbf{z}}\left(\mathbf{x}_{k}\right) d \mathbf{x}_{k} .
$$

\section{B. Standard SMC-PHD Filter}

The PHD filter can be implemented either as in the SMC fashion (particle-PHD) or as the GM-PHD. The SMC-PHD filter approximates the PHD using random samples and is more specifically an effective scheme in nonlinear and non-Gaussian scenarios as well as different noise models [35]. For comparison purposes, the standard SMC-PHD filter of [17] is briefly presented. The implementation of the standard SMC-PHD filter usually requires four stages. These stages are briefly presented in Algorithm 1.

Fig. 1 illustrates how particles are used to represent and track targets in the standard SMC-PHD filter. The state 


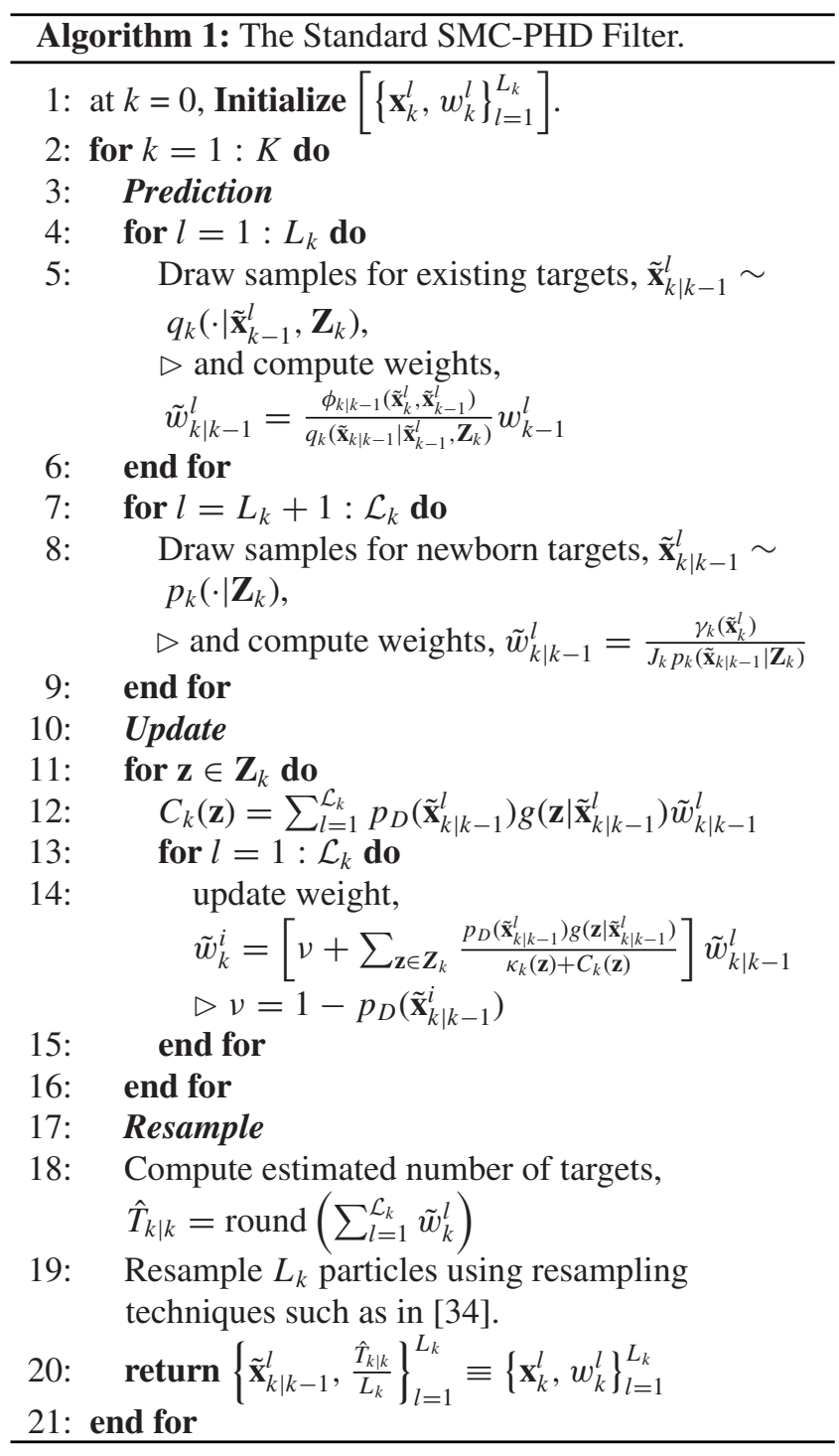

space of two targets populated with particles at time $k$ is shown. In A, during the prediction stage, the PHD is represented with eight equally weighted particles. In $\mathbf{B}$, as the latest measurement arrives, the particle weights are updated accordingly. Particles with higher weights are chosen for resampling. As seen in $\mathbf{B}$, the highly weighted particles are marked with " $\sqrt{ }$," respectively, five particles for the first target and six particles for the second target. To ensure that the number of particles remains eight for each target, the particles marked with " $\sqrt{ }$ " are resampled depending on the size of their weights as seen in $\mathbf{C}$. Notice that the particle positions remain unchanged and the particles corresponding to high weights are retained and those with lower weights are discarded. The estimated state of the targets or the posterior at time $k$ is derived from the resampled particles. It is true that populating the state space of the targets with many more particles will result in more particles falling near the modes of the state space. This will translate to higher weighted particles and a more accurate posterior. However, doing this will increase computational complexity.
In the next section, the proposed SMC-PHD filter is presented.

\section{PROPOSED SMC-PHD FILTER}

In the standard SMC-PHD filter, the particles appear to be scattered and it is difficult to guide particles to regions of interest. The filter's ability to estimate the posterior at a given time depends on how densely the state space is populated with samples and how well the estimated measurements match the actual measurements received in that time frame. The weights are then updated accordingly. The SMC-PHD filter does not provide for particle state correction to achieve particle improvement. In other words, it does not seek to reduce the error between the actual measurement and the estimated measurements irrespective of the importance density chosen. The proposed method seeks to address this problem. The novelty of our approach lies in the technique behind the Kalman filter. The Kalman filter is a minimum MSE estimator, which in effect seeks to recursively minimize the MSE between the estimated measurements and actual measurements using the Kalman gain [36]. The Kalman gain computes the required correction from the observation and transforms the correction of the observation back to the correction of state. The proposed approach tries to apply particle state correction/improvement using the Kalman gain to guide validated particles in the SMC-PHD filter to the region of higher likelihood to better approximate the posterior at each time step.

\section{A. Measurement Set Partition}

Given that $T_{k}$ targets exist at time $k$, the measurements received at $k$ may consist of target-originated measurements (i.e., measurements due to persistent target or newborn targets) and clutter. In the standard SMC-PHD filter, all measurements are used to compute weights to show the significance of all particles with no attempt to check for errors. Therefore, a measurement set partition is needed to separate the measurement set into targetoriginated measurements and measurements due to clutter. We use a statistical distance measure and gating technique to achieve this. The second step is to identify promising particles from the predicted target state using a validation threshold and improve their states using the Kalman gain while updating weights as measurement arrives.

At time $k$, measurements assumed to originate from persistent targets are identified by computing the square Mahalanobis distance between elements in the measurement set $\boldsymbol{Z}_{k-1}$ at time $k-1$ and $\boldsymbol{Z}_{k}$ at time $k$ from (3) as

$$
d_{i, j, k}^{2}=\left(\mathbf{z}_{k}^{i}-\mathbf{z}_{k-1}^{j}\right)^{T} \Sigma_{k}^{-1}\left(\mathbf{z}_{k}^{i}-\mathbf{z}_{k-1}^{j}\right)
$$

for $i=1, \ldots,\left|\boldsymbol{Z}_{k}\right|$ and $j=1, \ldots,\left|\boldsymbol{Z}_{k-1}\right| . \Sigma_{k}$ is the measurement covariance matrix. For target-originated measurements $\mathbf{z}_{k}^{i}$ and $\mathbf{z}_{k-1}^{j}$ belonging to the same target, the square Mahalanobis distance $d_{i, j, k}^{2}$ is $\chi^{2}$ distributed with degree of freedom equal to the dimension of the measurement vector. Therefore, a unit-less threshold $\tilde{d}$ can be computed for a 
given probability using the inverse cumulative $\chi^{2}$ function such that the $\operatorname{Pr}\left[d_{i, j, k}^{2} \leq \tilde{d}\right]$ falls within a given confidence region [3].

Assuming that measurement noise is not too great and the time increment is not too large, a target-generated measurement in $\boldsymbol{Z}_{k}$ will usually be nearby some measurement in $\boldsymbol{Z}_{k-1}$. Assuming that clutter is not too dense and is uncorrelated frame to frame, a clutter measurement in $\boldsymbol{Z}_{k}$ will usually not be near any measurement in $\boldsymbol{Z}_{k-1}$. Thus, $d_{i, j, k}^{2}$ as defined in (20) will tend to be small for some $j$ if $\mathbf{z}_{k}^{i}$ is due to a target. Likewise, it will tend to be large for all $j$ if $\mathbf{z}_{k}^{i}$ is a clutter measurement. So, for a given $i$, the measurement $\mathbf{z}_{k}^{i}$ is recorded as a valid target-originated measurement $\dot{\mathbf{z}}_{k}^{n}$ if

$$
\min _{j} d_{i, j, k}^{2} \leq \tilde{d}
$$

is satisfied or, otherwise, regarded either as clutter or a potential newborn target. Therefore, the clutter-free measurement set at time $k$ is

$$
\dot{\mathbf{Z}}_{k}=\bigcup_{n=1}^{n_{s}}\left\{\dot{\mathbf{z}}_{k}^{n}\right\}
$$

where $n=1, \ldots, n_{s}$ and $n_{s}=\left|\dot{\boldsymbol{Z}}_{k}\right|$, that is, the total number of measurements in $Z_{k}$ satisfying (21).

\section{B. Validated Particle Selection and Correction}

Once the measurement set is partitioned, the selection and correction step follows. In order to identify those particles to correct, a validation threshold $\tau$ is used, which selects particles from the predicted target state that fall under a given measurement for correction. A predicted particle, $\tilde{\mathbf{x}}_{k \mid k-1}^{l}$ is selected for correction if, for each clutter-free measurement $\dot{\mathbf{z}}_{k}^{n} \in \dot{\boldsymbol{Z}}_{k}$

$$
g\left(\dot{\mathbf{z}}_{k}^{n} \mid \tilde{\mathbf{x}}_{k \mid k-1}^{l}\right) \geq \tau, \quad \text { for } l=1, \ldots, \mathcal{L}_{k}
$$

where $g\left(\dot{\mathbf{z}}_{k}^{n} \mid \tilde{\mathbf{x}}_{k \mid k-1}^{l}\right)$ is the measurement likelihood function; $\tau$ is chosen to be inversely proportional to the total number of samples per persistent target $\rho$, i.e.,

$$
\tau \propto \frac{1}{\rho} .
$$

Each particle satisfying (23) is assumed to be a reasonable candidate for correction given the current measurement. A large $\tau$ will lead to a tighter particle selection while a smaller value of $\tau$ will result in wider particle selection, that is, more particles will be selected for correction. Once a reasonable candidate $\tilde{\mathbf{x}}_{k \mid k-1}^{l}$ has been identified, it's state is corrected as

$$
\begin{aligned}
\tilde{\mathbf{x}}_{k}^{l} & =\tilde{\mathbf{x}}_{k \mid k-1}^{l}+\mathcal{K}_{k}\left(\dot{\mathbf{z}}_{k}^{n}-f\left(\tilde{\mathbf{x}}_{k \mid k-1}^{l}\right)\right) \\
\mathcal{K}_{k} & =\mathbf{P}_{k-1} \mathbf{H}_{k}^{T} \mathbf{S}_{k}^{-1} \\
\mathbf{S}_{k}^{-1} & =\mathbf{R}_{k}+\mathbf{H}_{k} \mathbf{P}_{k-1} \boldsymbol{H}_{k}^{T} \\
\mathbf{P}_{k} & =\mathbf{P}_{k-1}-\mathcal{K}_{k} \mathbf{H}_{k} \mathbf{P}_{k-1}
\end{aligned}
$$

where $f\left(\tilde{\mathbf{x}}_{k \mid k-1}^{l}\right)$ is the projection of the predicted state $\tilde{\mathbf{x}}_{k}^{l}$ on to the measurement, $\mathcal{K}_{k}$ is the Kalman gain, $\mathbf{H}$ is the measurement transformation matrix, $\mathbf{P}$ is the state estimation covariance matrix, $\mathbf{R}$ is the measurement error covariance matrix, and $\mathbf{S}$ is the innovation covariance matrix.

Therefore, given that the $t$ th target generated the clutterfree measurement $\dot{\mathbf{z}}_{t, k}$ at time $k$, and its state is represented by particles $\left\{\mathbf{x}_{t, k}\right\}_{t=1}^{\rho, k}$ from the predicted target state, then, only those particles, $\left\{\mathbf{x}_{t, k}\right\}_{t=1}^{s}$ satisfying (23) will be selected for correction according to (25) where $s \leq \rho$.

Fig. 2 illustrates how particles representing the state of the PHD of targets are selected for correction as the measurement originating from the $t$ th target arrives at time $k$. From the figure, in $\mathbf{A}$, during the prediction stage, each of the target states is represented with eight equally weighted particles. As the latest measurement for each target arrives, particles with high likelihood are marked with " $\sqrt{ }$ " as seen in $\mathbf{B}$. The validation threshold $\tau$ of (23) is then applied to the likelihood of particles with " $\sqrt{ }$." Each particle whose likelihood satisfies the threshold condition is selected for correction. The selected particles are shown with “*” in $\mathbf{B}$. The particle weights are updated accordingly. Notice from B that for the first target, five particles have high likelihood but only three from the five were chosen for state correction. Similarly, for the second target, six particles gave high likelihood but out of which only four were selected for state correction. The selected particles from $\mathbf{B}$ are then corrected using (25) and (26) as shown in $\mathbf{C}$. The corrected particles are then resampled to ensure that the number of particles remains eight for each target.

Note, the above measurement set partition approach can be applied under the following assumptions: that newborn targets exist for at least two consecutive time steps, the maneuvering of targets is not too abrupt, the sample period $\delta t$ is not too large, measurement noise is not too large, clutter is not too dense, and clutter is not time correlated.

Furthermore, we emphasize that the proposed KGSMC-PHD is different from the GM-PHD in [16] even with the application of the gating technique as will be demonstrated in the simulation section. This is primarily because we do not assume the strict linearity and Gaussianity condition of [16].

\section{KG-SMC-PHD Implementation of the PHD Filter}

We now present the initialization, prediction, update, and resample steps of the KG-SMC-PHD filter.

1) Initialization: At time $k=0$, initialize the PHD $D_{k \mid k}$ by a number of particles with associated weights $\left\{\mathbf{x}_{k}^{l}, w_{k}^{l}\right\}_{l=1}^{L_{k}}$. A particle approximation of the intensity function at time step $k>0$ can be obtained from a particle distribution at the previous time step using prediction and update stages.

2) Prediction: The predicted PHD $D_{k \mid k-1}$ is

$$
D_{k \mid k-1}\left(\tilde{\mathbf{x}}_{k} \mid \dot{\mathbf{Z}}_{k-1}\right)=\sum_{l=1}^{\mathcal{L}_{k}} \tilde{w}_{k \mid k-1}^{l} \delta\left(\mathbf{x}-\tilde{\mathbf{x}}_{k \mid k-1}^{l}\right) .
$$

We draw $L_{k-1}$ and $J_{k}$ particles from two proposal densities (chosen from the possibilities discussed in Section III, i.e., TP, EPF or UPF) to represent persistent and newborn 


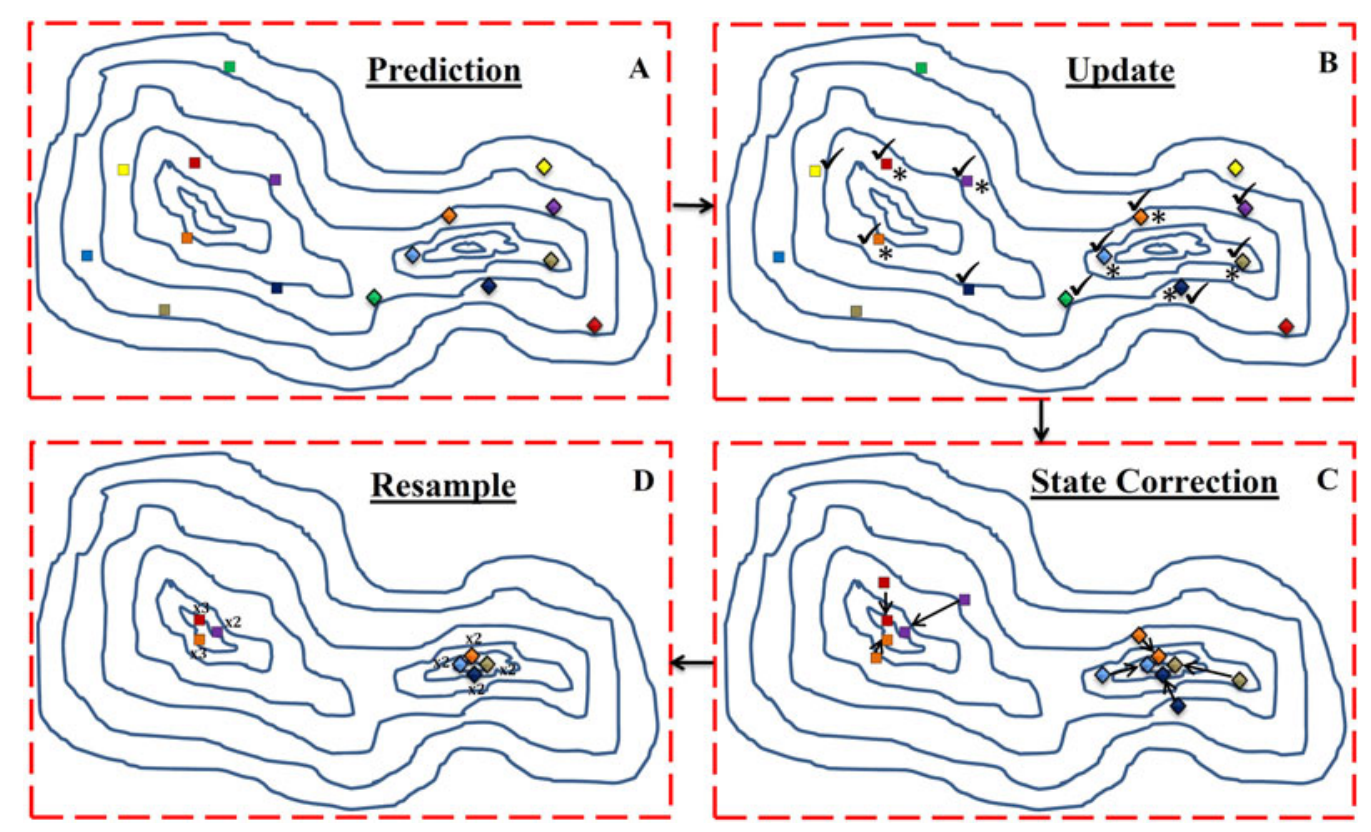

Fig. 2. Schematic representation of the proposed SMC-PHD filter showing the 2-D state space of the PHD of two targets populated with particles. The contours represent the state space of targets. The contour centers and number of centers represent the mode and number of targets, respectively. Boxes $\mathbf{A}, \mathbf{B}, \mathbf{C}$, and $\mathbf{D}$ represent various stages of the filter. The square-shaped and diamond-shaped particles are for target 1 and target 2 , respectively.

The colors stand for different particle states. The particles with " $\sqrt{ }$ " stand for particles with higher weight for when latest the observation arrives. Particles with “*” denote particles selected for state correction.

targets, respectively, as

$$
\tilde{\mathbf{x}}_{k \mid k-1}^{l} \approx \begin{cases}q_{k}\left(\cdot \mid \tilde{\mathbf{x}}_{k-1}^{l}, \dot{\mathbf{Z}}_{k}\right), & l=1, \ldots, L_{k-1} \\ p_{k}\left(\cdot \mid \dot{\mathbf{Z}}_{k}\right), & l=L_{k-1}+1, \ldots, \mathcal{L}_{k}\end{cases}
$$

with corresponding weights

$$
\tilde{w}_{k \mid k-1}^{l}= \begin{cases}\frac{\phi_{k \mid k-1}\left(\tilde{\mathbf{x}}_{k}^{l}, \tilde{\mathbf{x}}_{k-1}^{l}\right)}{q_{k}\left(\tilde{\mathbf{x}}_{k \mid k-1} \mid \tilde{\mathbf{x}}_{k-1}^{l}, \mathbf{Z}_{k}\right)} w_{k-1}^{l}, & l=1, \ldots, L_{k-1} \\ \frac{\gamma_{k}\left(\tilde{\mathbf{x}}_{k}^{l}\right)}{J_{k} p_{k}\left(\tilde{\mathbf{x}}_{k \mid k-1} \mid \mathbf{Z}_{k}\right)}, & l=L_{k-1}+1, \ldots, \mathcal{L}_{k}\end{cases}
$$

with the term

$$
\begin{aligned}
\phi_{k \mid k-1}\left(\mathbf{x}_{k}, \mathbf{x}_{k-1}\right)= & p_{S}\left(\mathbf{x}_{k-1}\right) f_{k \mid k-1}\left(\mathbf{x}_{k}, \mathbf{x}_{k-1}\right) \\
& +b_{k \mid k-1}\left(\mathbf{x}_{k}, \mathbf{x}_{k-1}\right)
\end{aligned}
$$

where $\mathcal{L}_{k}=L_{k-1}+J_{k}, q_{k}(\cdot \mid \cdot)$ and $p_{k}(\cdot \cdot \cdot)$ denote the proposal distributions for persistent and newborn targets, respectively; $\gamma_{k}(\cdot)$ is the PHD of the spontaneous birth, $p_{S}(\cdot)$ is the probability of target survival, $f_{k \mid k-1}\left(\mathbf{x}_{k}, \mathbf{x}_{k-1}\right)$ is the single target motion model, and $b_{k \mid k-1}\left(\mathbf{x}_{k}, \mathbf{x}_{k-1}\right)$ is the PHD of spawned targets; $J_{k}$ is the number of particles for newborn targets.

3) Update: For each $\dot{\mathbf{z}}_{k}^{n} \in \dot{\mathbf{Z}}_{k}$ where $\dot{\mathbf{Z}}_{k}$ is the clutterfree measurement set at time $k$ obtained using (20) and (21), let

$$
\begin{aligned}
\mathcal{H}\left(\dot{\mathbf{z}}_{k}^{n}\right) & =\kappa\left(\dot{\mathbf{z}}_{k}^{n}\right)+C_{k}\left(\dot{\mathbf{z}}_{k}^{n}\right) \\
C_{k}\left(\dot{\mathbf{z}}_{k}^{n}\right) & =\sum_{l=1}^{\mathcal{L}_{k}} p_{D}\left(\tilde{\mathbf{x}}_{k \mid k-1}^{l}\right) g\left(\dot{\mathbf{z}}_{k}^{n} \mid \tilde{\mathbf{x}}_{k \mid k-1}^{l}\right) \tilde{w}_{k \mid k-1}^{l}
\end{aligned}
$$

then, for $l=1, \ldots, \mathcal{L}_{k}$, compute the likelihood $g\left(\dot{\mathbf{z}}_{k}^{n} \mid \tilde{\mathbf{x}}_{k \mid k-1}^{l}\right)$ and verify if (23) is true, correct predicted state by computing $\tilde{\mathbf{x}}_{k}^{l}$ using (25) then compute (33) and update the weights using

$$
\tilde{w}_{k}^{l}=\left[v+\sum_{\dot{\mathbf{z}} \in \dot{\mathbf{Z}}_{k}} \frac{p_{D}\left(\tilde{\mathbf{x}}_{k \mid k-1}^{l}\right) g\left(\dot{\mathbf{z}}_{k}^{n} \mid \tilde{\mathbf{x}}_{k \mid k-1}^{l}\right)}{\mathcal{H}\left(\dot{\mathbf{z}}_{k}^{n}\right)}\right] \tilde{w}_{k \mid k-1}^{l}
$$

where $v=1-p_{D}\left(\tilde{\mathbf{x}}_{k \mid k-1}^{l}\right)$. However, if (23) is not satisfied, the predicted state is not corrected; $\tilde{\mathbf{x}}_{k}^{l}$ is computed as $\tilde{\mathbf{x}}_{k}^{l}=$ $\tilde{\mathbf{x}}_{k \mid k-1}^{l}$ and (32) and (34) are computed immediately.

The updated PHD, $D_{k \mid k}$ is then given as

$$
D_{k \mid k}\left(\tilde{\mathbf{x}}_{k} \mid \dot{\mathbf{Z}}_{k}\right)=\sum_{l=1}^{\mathcal{L}_{k}} \tilde{w}_{k}^{l} \delta\left(\mathbf{x}-\tilde{\mathbf{x}}_{k}^{l}\right) .6
$$

\section{4) Resample:}

1) The expected number of targets $\hat{T}_{k \mid k}$ is computed as

$$
\hat{T}_{k \mid k}=\operatorname{round}\left(\sum_{l=1}^{\mathcal{L}_{k}} \tilde{w}_{k}^{l}\right)
$$

where round $(\cdot)$ denotes round to the nearest integer.

2) $L_{k}=\rho \hat{T}_{k \mid k}$ particles are resampled ( $\rho$ corresponds to the number of particles per existing target) according to the modified systematic resampling technique below.

a) Find all noncontributing weights $\bar{w}_{k}$ from $\tilde{w}_{k}$ such that $\bar{w}_{k} \in \tilde{w}_{k}$ and replace with $\varpi$, where $0<\varpi \ll$ $\frac{1}{\rho}$. This is to ensure that only weights belonging to corrected particles are chosen for resampling. 


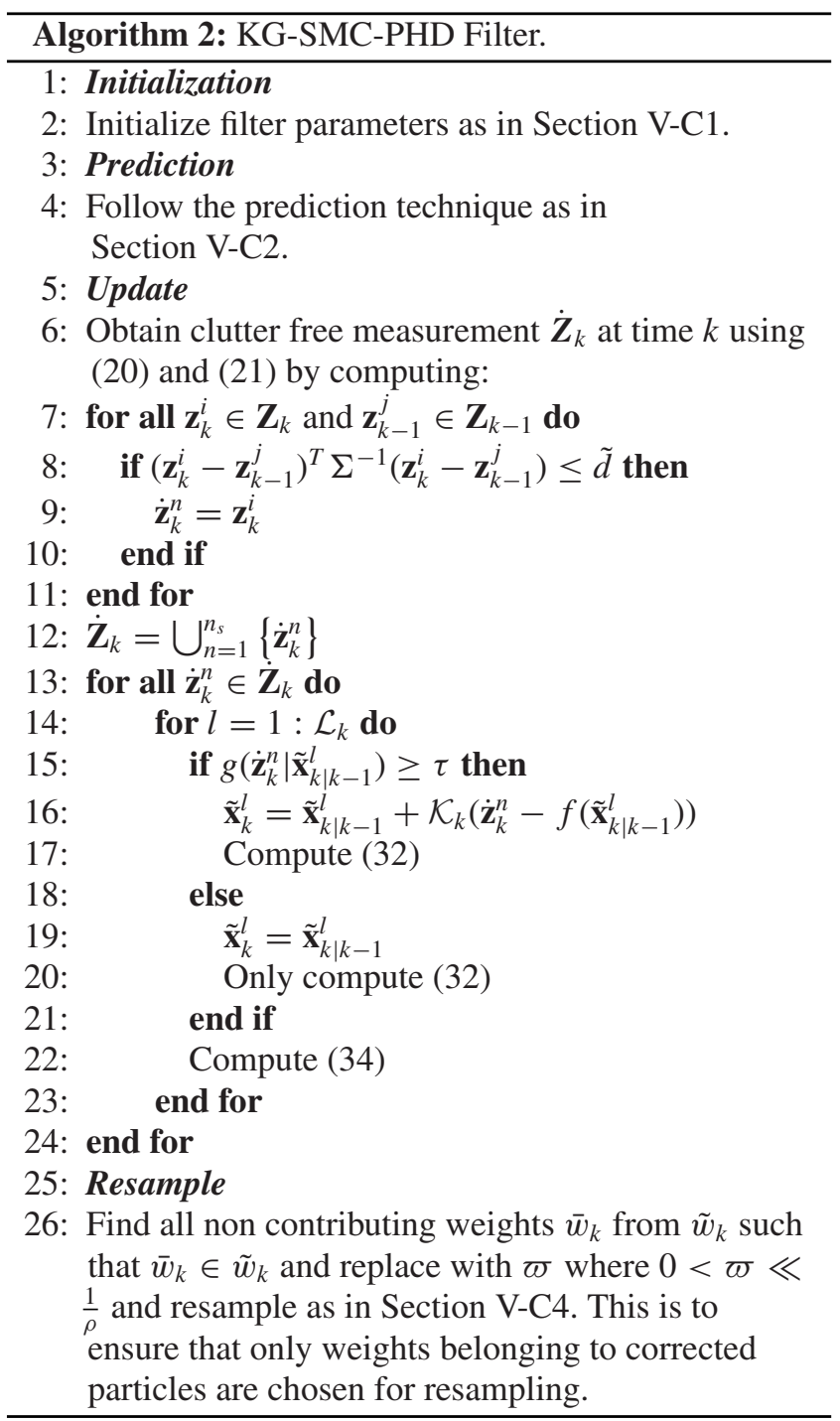

b) Then compute cumulative probability $c_{1}=0, c_{l}=$ $c_{l-1}+\left(\frac{\tilde{w}_{k}^{l}}{\hat{T}_{k \mid k}}\right), l=2, \ldots, L_{k}+J_{k}$.

c) Draw a starting point $u_{1}$ from $U\left[0, \frac{1}{L_{k}}\right]$.

d) For $j=1, \ldots, L_{k}$

$$
u_{j}=u_{1}+L_{k}^{-1}(j-1)
$$

while $u_{j}>c_{i}, l=l+1$. End while

$$
\begin{aligned}
\mathbf{x}_{k}^{j} & =\tilde{\mathbf{x}}_{k}^{j} \\
w_{k}^{j} & =\frac{1}{L_{k}} .
\end{aligned}
$$

3) Rescale (multiply) the weights by $\hat{T}_{k \mid k}$ to obtain $\left\{\mathbf{x}_{k}^{l}, \frac{\hat{k}_{k \mid k}}{L_{k}}\right\}_{l=1}^{L_{k}}$, where $w_{k}^{l}=\frac{\hat{T}_{k \mid k}}{L_{k}}$ therefore $\left\{\mathbf{x}_{k}^{l}, \frac{\hat{T}_{k \mid k}}{L_{k}}\right\}_{l=1}^{L_{k}} \equiv$ $\left\{\mathbf{x}_{k}^{l}, w_{k}^{l}\right\}_{l=1}^{L_{k}}$.

The pseudocode of the proposed KG-SMC-PHD filter is described in Algorithm 2.

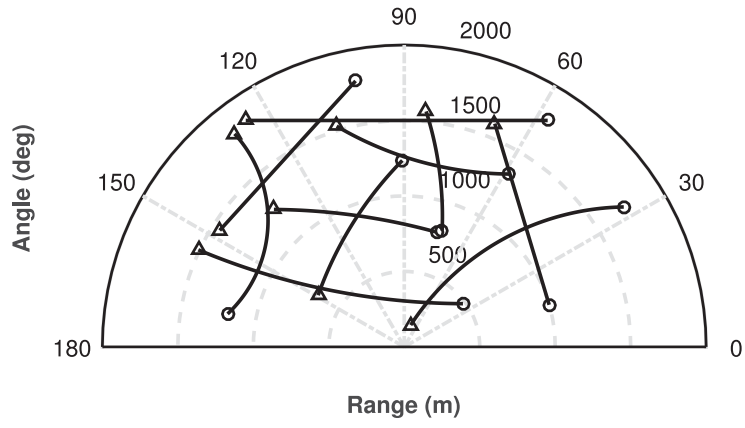

Fig. 3. True target trajectories in the range-bearing plane with start/end positions for each track shown with $\bigcirc / \triangle$

\section{SIMULATION RESULTS}

In this section, the nonlinear tracking performance of the proposed KG-SMC-PHD filter is demonstrated.

\section{A. Simulation Context and Filter Parameters}

We consider a two-dimensional (2-D) nonlinear range and bearing scenario with unknown and varying number of targets observed over a cluttered region. A total of ten targets enter and exit the scene at various times throughout the simulation scenario. The observation region is a half disk of radius $2000 \mathrm{~m}$. A plot of the ground truth (true trajectories) of the targets along with the start and end positions of each track is shown in Fig. 3. The start and end positions are indicated by a circle and a triangle, respectively. The nonlinear target dynamics are described by a nearly constant turn state model driven by white noise acceleration

$$
\hat{\mathbf{x}}_{k}=\mathbf{F}\left(\omega_{k-1}\right) \hat{\mathbf{x}}_{k-1}+\Gamma \mathbf{v}_{k}
$$

$$
\omega_{k}=\omega_{k-1}+\delta t u_{k-1}
$$

where

$$
\begin{aligned}
\mathbf{F}(\omega) & =\left[\begin{array}{cccc}
1 & \frac{\sin \omega \delta t}{\omega} & 0 & -\frac{1-\cos \omega \delta t}{\omega} \\
0 & \cos \omega \delta t & 0 & -\sin \omega \delta t \\
0 & \frac{1-\cos \omega \delta t}{\omega} & 1 & \frac{\sin \omega \delta t}{\omega} \\
0 & \sin \omega \delta t & 0 & \cos \omega \delta t
\end{array}\right], \\
\Gamma & =\left[\begin{array}{cc}
\frac{\delta t^{2}}{2} & 0 \\
\delta t & 0 \\
0 & \frac{\delta t^{2}}{2} \\
0 & \delta t
\end{array}\right] .
\end{aligned}
$$

$\mathbf{F}(\omega)$ is the transition matrix for nearly constant turn rate, $\delta t$ denotes the sample period which is assumed to be $1 s$ in this simulation, and $\Gamma$ denotes the input matrix. The target state vector $\mathbf{x}_{k}=\left[\hat{\mathbf{x}}_{k}, \omega_{k}\right]^{\bar{T}}$ comprises the planar positions and velocities given as $\hat{\mathbf{x}}_{k}=\left[x_{k}, \dot{x}_{x}, y_{k}, \dot{y}_{k}\right]^{\bar{T}}$ along with turn rate $\omega_{k}$. The variables $\left(x_{k}, y_{k}\right)$ represent the 
position of the target and $\left(\dot{x}_{k}, \dot{y}_{k}\right)$ represent the velocities. $\mathbf{v}_{k}=\mathcal{N}\left(\cdot, 0, \sigma_{v}^{2} I\right)$ and $u_{k-1}=\mathcal{N}\left(\cdot, 0, \sigma_{u}^{2} I\right)$ with $\sigma_{v}=$ $10 \mathrm{~m} / \mathrm{s}^{2}$ and $\sigma_{u}=\pi / 180 \mathrm{rad} / \mathrm{s}$. [. $]^{\bar{T}}$ denotes transpose operation.

Targets can appear and disappear in the tracking volume at any time. Target spawning is not considered in this example. Each persistent target has a probability of survival, $p_{S}\left(\mathbf{x}_{k-1}\right)=0.9$. The target birth process is modeled as a Poisson point process with intensity function $\quad \gamma_{k}\left(\mathbf{x}_{k}\right)=0.3 \mathcal{N}\left(\cdot, \dot{\mathbf{x}}_{1}, \mathbf{Q}\right)+0.3 \mathcal{N}\left(\cdot, \dot{\mathbf{x}}_{2}, \mathbf{Q}\right)+$ $0.3 \mathcal{N}\left(\cdot, \mathbf{x}_{3}, \mathbf{Q}\right)+0.3 \mathcal{N}\left(\cdot, \dot{\mathbf{x}}_{4}, \mathbf{Q}\right)+0.3 \mathcal{N}\left(\cdot, \dot{\mathbf{x}}_{5}, \mathbf{Q}\right)$, where $\dot{\mathbf{x}}_{1}=[-1000,0,200,0]^{\bar{T}}, \dot{\mathbf{x}}_{2}=[1000,0,1500,0]^{\bar{T}}, \mathbf{x}_{3}=$ $[0,0,1500,0]^{\bar{T}}, \quad \dot{\mathbf{x}}_{4}=[500,0,500,0]^{\bar{T}}, \quad \dot{\mathbf{x}}_{5}=[1500,0$, $1000,0]^{\bar{T}}$ and covariance matrix, $\mathbf{Q}=\operatorname{diag}([200,50,200$, $\left.50,6(\pi / 180)]^{\bar{T}}\right)$.

The target-originated measurements are given by the nonlinear model

$$
\boldsymbol{z}_{k}=\left[\begin{array}{c}
r_{k} \\
\theta_{k}
\end{array}\right]+\mathbf{n}_{k}
$$

with

$$
r_{k}=\left\|\left[\begin{array}{llll}
1 & 0 & 0 & 0 \\
0 & 0 & 1 & 0
\end{array}\right] \mathbf{x}_{k}-\left[\begin{array}{l}
x_{s} \\
y_{s}
\end{array}\right]\right\|
$$

and

$$
\theta_{k}=\arctan \left(\frac{\left[\begin{array}{llll}
0 & 0 & 1 & 0
\end{array}\right] \mathbf{x}_{k}+y_{s}}{\left[\begin{array}{llll}
1 & 0 & 0 & 0
\end{array}\right] \mathbf{x}_{k}+x_{s}}\right)
$$

where the measurement noise $\mathbf{n}_{k}$ is a zero-mean Gaussian white noise vector with covariance matrix $\mathbf{R}=$ $\operatorname{diag}\left(\left[\sigma_{r}^{2}, \sigma_{\theta}^{2}\right]\right)$ with $\sigma_{r}=10 \mathrm{~m}$ and $\sigma_{\theta}=0.5 \mathrm{rad}$. The measurement sensor's location, $\left[x_{s}, y_{s}\right]^{T}$ is at the origin. Clutter is uniformly distributed over the observation region of $[0, \pi] \times[0,2000]$ with a Poisson point process on the clutter region with a uniform intensity function $\kappa_{k}=3.2 \times 10^{-3}(\mathrm{radm})^{-1}$ (giving an average of $\lambda=20$ clutter points per scan). The total number of particles at time $k$ is

$$
\mathcal{L}_{k}=L_{k}+J_{k} \quad \text { and } \quad L_{k} \cong \rho \hat{T}_{k \mid k}
$$

where $\hat{T}_{k \mid k}$ denotes the expected number of targets, $L_{k}$ is the number of particles for all persistent tracks, $\rho$ denotes number of particles per persistent track, and $J_{k}=\frac{\rho}{5}$ is the number of samples per newborn track. The probability of detection $p_{D}\left(\mathbf{x}_{k}\right)$ is 0.9 .

To analyze the estimation error of the filter, we use the optimal subpattern assignment (OSPA) proposed in [37] and computation time (CT). The OSPA distance metric enables us to compare multitarget filtering algorithms [37]. The OSPA distance between two arbitrary finite sets, that is, the state set $\mathbf{A}=\left\{\mathbf{a}_{1}, \ldots, \mathbf{a}_{m}\right\}$ and the ground truth state set $\mathbf{B}=\left\{\mathbf{b}_{1}, \ldots, \mathbf{b}_{n}\right\}$ is

$$
\bar{d}_{\dot{p}}^{(\dot{c})}(\mathbf{A}, \mathbf{B})= \begin{cases}0, & \text { if } \quad \dot{m}=\dot{n}=0 \\ \Theta(\mathbf{A}, \mathbf{B}), & \text { if } \quad \dot{m} \leq \dot{n} \\ d^{(\dot{c})}(\mathbf{A}, \mathbf{B}), & \text { if } \quad \dot{m}>\dot{n}\end{cases}
$$

TABLE I

Filter Performance Comparison in Terms of OSPA Distance, CT, and PD for $\lambda=20$ and $\rho=1000$ with Measurement Partition

\begin{tabular}{lccc}
\hline \hline Filter & PD & OSPA (m) & CT (s) \\
\hline SMC-PHD & TP (from Section III-B1) & 94.85 & 9.50 \\
& EPF (from Section III-B2) & 83.17 & 11.67 \\
KG-SMC-PHD & UPF (from Section III-B3) & 81.72 & 12.85 \\
& TP (from Section III-B1) & 22.19 & 10.43 \\
& EPF (from Section III-B2) & 19.64 & 13.40 \\
& UPF (from Section III-B3) & 18.69 & 14.51 \\
\hline
\end{tabular}

TABLE II

Filter Performance Comparison in Terms of OSPA Distance, CT, and PD for $\lambda=30$ and $\rho=1000$ with Measurement Partition

\begin{tabular}{lccc}
\hline \hline Filter & PD & OSPA (m) & CT (s) \\
\hline SMC-PHD & TP (from Section III-B1) & 111.76 & 10.63 \\
& EPF (from Section III-B2) & 105.46 & 12.15 \\
\multirow{3}{*}{ KG-SMC-PHD } & UPF (from Section III-B3) & 103.78 & 13.40 \\
& TP (from Section III-B1) & 31.56 & 11.77 \\
& EPF (from Section III-B2) & 21.70 & 14.90 \\
& UPF (from Section III-B3) & 20.15 & 15.76
\end{tabular}

where

$\Theta(\mathbf{A}, \mathbf{B}) \triangleq\left(\frac{1}{\dot{n}}\left(\min _{\pi \in \prod_{\tilde{n}}} \sum_{i=1}^{\dot{m}} d^{(\hat{c})}\left(a_{i}, b_{\pi(i)}\right)^{\dot{p}}+\dot{c}^{\dot{p}}(\dot{n}-\dot{m})\right)\right)^{\frac{1}{\hat{p}}}$.

$\prod_{n}$ is the set of permutations with length $\dot{m}$ on the set

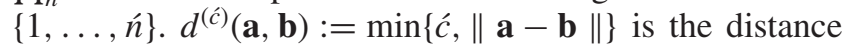
between single target vectors a and $\mathbf{b}$. $\dot{c}>0$ is the cut-off parameter and $\dot{p} \geq 1$ is a unit-less real number. We choose parameters $\dot{c}=300$ and $\dot{p}=1$. The cut-off parameter $\dot{c}$ determines the relative weighting of the penalties assigned to localization and cardinality errors, and $\dot{p}$ determines the sensitivity to outliers. For more details on the OSPA metric, the reader is referred to [37].

\section{B. Effect of Proposal Distributions}

Here, different importance sampling functions, TP, EPF, and UPF of Section III are applied to the SMC-PHD and KG-SMC-PHD filters to observe the effects of each choice on filter performance. The results obtained are shown in Tables I and II. The number of particles used in each of the two clutter cases is $\rho=1000$. Tables I and II show results averaged over $1000 \mathrm{MC}$ trials for $\lambda=20$ and $\lambda=30$ with measurement set partition, respectively. Overall, using the UPF as a proposal distribution gives better performance for both filters in terms of low OSPA distance but this method incurs the most computational load. This is primarily due to the generation of sigma points for each particle and the computation that follows during the unscented transform process. Using both EPF and UPF to construct the proposal distributions give better performance in terms of yielding lower OSPA, when compared to using TP. This is because both EPF and UPF helps us to place generated samples "under" measurements as soon as measurements become available. However, the improvement of using the UPF over 
TABLE III

Filter Performance in Terms of Number of Particles, OSPA Distance, and CT for $\lambda=20$ with Measurement Partition

\begin{tabular}{lccc}
\hline \hline Filter & $\rho$ & OSPA $(\mathrm{m})$ & CT (s) \\
\hline SMC-PHD & 50 & 149.31 & 0.42 \\
& 100 & 100.36 & 0.86 \\
& 500 & 86.38 & 5.19 \\
& 1000 & 83.17 & 11.67 \\
KG-SMC-PHD & 50 & 44.70 & 0.72 \\
& 100 & 33.29 & 1.26 \\
& 500 & 22.11 & 6.29 \\
& 1000 & 19.64 & 13.40 \\
\hline
\end{tabular}

TABLE IV

Filter Performance in Terms of Number of Particles, OSPA Distance, and CT for $\lambda=30$ with Measurement Partition

\begin{tabular}{lccc}
\hline \hline Filter & $\rho$ & OSPA (m) & CT (s) \\
\hline SMC-PHD & 50 & 154.82 & 0.47 \\
& 100 & 117.88 & 0.93 \\
& 500 & 106.19 & 5.99 \\
KG-SMC-PHD & 50 & 47.15 & 0.87 \\
& 1000 & 39.30 & 1.36 \\
& 500 & 25.79 & 7.02 \\
& 1000 & 21.70 & 14.90 \\
\hline
\end{tabular}

EPF is not too significant in terms of OSPA distance. As a result, the EPF will be used as the importance sampling function for both filters in our subsequent discussion.

\section{Varying Number of Particles}

For this case, the EPF was chosen as the importance sampling density for both filters. This is because as discussed earlier, using the EPF gives a lower CT. Tables III and IV show results of filter performance in terms of number of particles, OSPA distance, and CT obtained for both filters averaged over $1000 \mathrm{MC}$ simulations for different $\rho$ values when clutter is present with measurement partition. It can be observed from both tables that the performance of the SMC-PHD filter appears to deteriorate further with more position and cardinality mismatch (high OSPA distance) as clutter density increases while the proposed filter is seen to maintain a consistent performance with improved accuracy in position and cardinality (low OSPA distance). The CT of the proposed filter, however, is seen to be higher than the SMC-PHD filter for a given $\rho$ value. This is due to the particle state correction step of the proposed filter. However, the number of particles required in terms of performance level (i.e., low OSPA) by the proposed filter is far less when compared to the SMC-PHD filter making the proposed filter more efficient.

Fig. 4 depicts the average of $1000 \mathrm{MC}$ runs of the true and estimated number of targets for $\rho=500$ with average number of clutter per scan, $\lambda=20$. This result shows that the KG-SMC-PHD filter is able to estimate properly the number of targets under such high clutter condition. Fig. 5

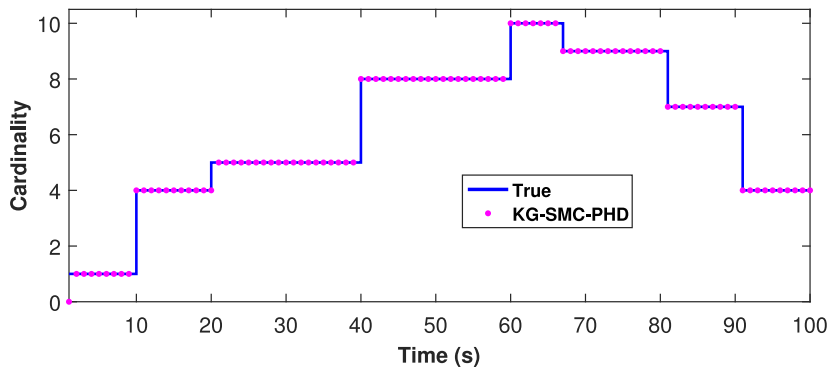

Fig. 4. True and KG-SMC-PHD filter cardinality estimates of targets time averaged over $1000 \mathrm{MC}$ trials with $\rho=500$ particles per existing track.

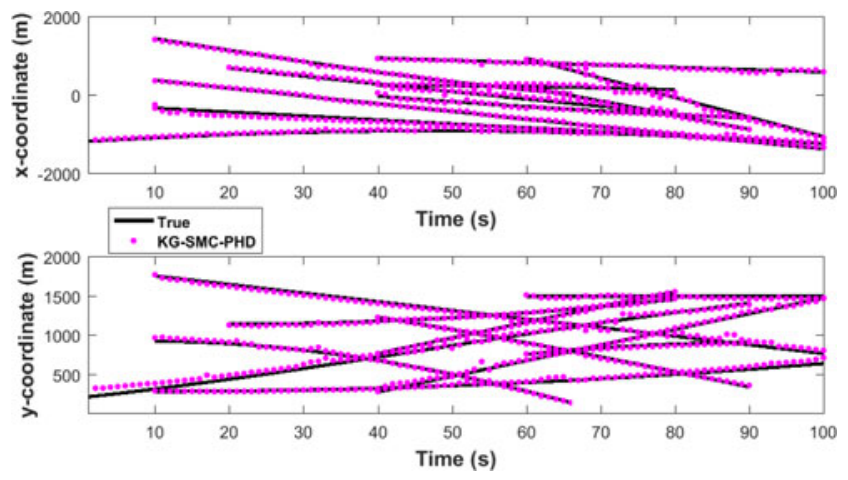

Fig. 5. $x$ and $y$ components (versus time) of the true target trajectories and the KG-SMC-PHD filter estimates for $\rho=500$ particles per existing track.

shows the $x$ and $y$ components (versus time) of the true trajectories and the KG-SMC-PHD filter estimates. The plots indicate that the proposed filter with $\rho=500$ particles per existing track is able to properly track all targets and in addition to being able to identify all target births and deaths while successfully accommodating nonlinearities under high clutter condition.

\section{Other Filters}

To further demonstrate the performance of the KGSMC-PHD filter, the proposed filter was evaluated along with the GM-PHD filter of [16], the GM-USMC-PHD filter of [21], and the AP-PHD filter in [23] in addition to the standard SMC-PHD filter. The evaluation is in terms of OSPA distance and CT. For this comparison, the EPF was used to construct the importance sampling function for both the KG-SMC-PHD filter and the SMC-PHD filter. The KGSMC-PHD filter was evaluated at 500 and $\frac{500}{5}$ particles for existing and newborn tracks, respectively, while 1000 and $\frac{1000}{5}$ particles for existing and newborn tracks, respectively, were used for the SMC-PHD filter.

The GM-PHD filter was implemented with an EKF. The maximum number of Guassian terms was set to 100 , with the merging $\left(T_{m}\right)$ and pruning threshold $\left(T_{p}\right)$ set at $10 \mathrm{~m}$ and $10^{-3}$, respectively. A Gaussian component is considered to be target originated if its weight is above 0.4 . The estimated number of targets is given by the sum of weights of the GM. 
TABLE V

Filter Performance Comparison in Terms of OSPA Distance and CT for $\lambda=20$ with Measurement Set Partition

\begin{tabular}{lcc}
\hline \hline Filter & OSPA (m) & CT (s) \\
\hline KG-SMC-PHD & 22.11 & 6.29 \\
SMC-PHD & 83.17 & 11.67 \\
GM-PHD & 38.05 & 2.59 \\
GM-USMC-PHD & 35.38 & 13.58 \\
AP-PHD & 33.19 & 16.99
\end{tabular}

TABLE VI

Filter Performance Comparison in Terms of OSPA Distance and CT for Different Filters for $\lambda=20$ without Measurement Set Partition

\begin{tabular}{lcc}
\hline \hline Filter & OSPA (m) & CT (s) \\
\hline KG-SMC-PHD & 22.11 & 6.29 \\
SMC-PHD & 94.66 & 10.11 \\
GM-PHD & 44.93 & 1.90 \\
GM-USMC-PHD & 47.23 & 12.25 \\
AP-PHD & 39.01 & 14.22 \\
\hline
\end{tabular}

In the AP-PHD filter implementation, 1000 particles were used per existing track and $\frac{1000}{5}$ particles were used for the newborn track. Each new track initialization is measurement driven and each current measurement is associated with the corresponding highest bidder if the bid is greater than 0.4. The auxiliary importance sampling [23] process starts with the selection of the measurements that are well described by the targets states extracted from the estimated PHD and this is achieved using the auction algorithm. Both auctioning and state extraction is done as in [23].

The GM-USMC-PHD filter uses a GM to approximate the importance density function. The GM implementation of the GM-USMC-PHD filter is similar to the GM-PHD filter in terms of number of Gaussian components, and pruning and merging thresholds. The number of samples per GM component is set to 1000 . The newborn track initialization, resampling, and state extraction steps follow [21] and the mean and the covariance of Gaussian is computed using the UIF [3].

Tables V and VI show filter performance results averaged over $1000 \mathrm{MC}$ runs with and without measurement set partition, respectively. In both tables, measurement partition was applied to the KG-SMC-PHD filter. The effect of the measurement partitioning process can be seen in Table $\mathrm{V}$ as the OSPA distance improved for the other filters. This is because measurements due to clutter were discarded and not used in the weight update stages of the filters. Notice also there is a slight increase in CT from Table V as compared to VI. This reflects the added CT during the partitioning process. Overall, under high clutter, the KGSMC-PHD filter gives a better performance as it maintains low OSPA distance. This is mainly due to our particle state

\footnotetext{
${ }^{2}$ The OSPA [37] metric measures the combination of both localization and cardinality distance.
}

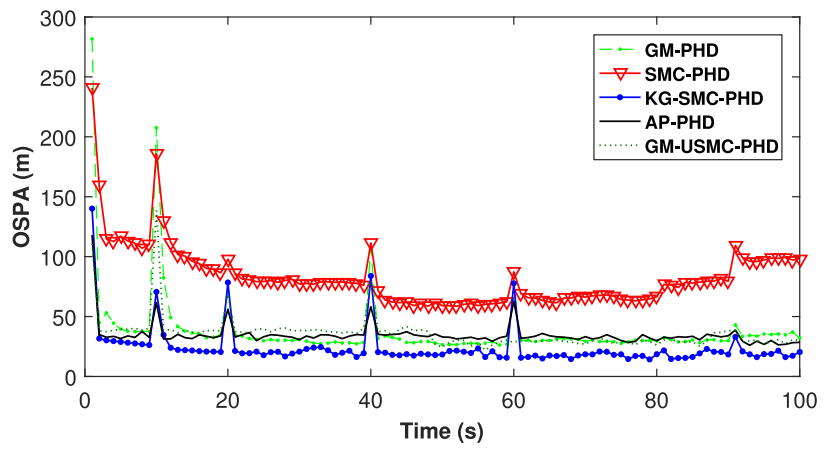

Fig. 6. OSPA distance averaged over $1000 \mathrm{MC}$ runs for clutter rate of 20 Poisson clutter $(\lambda=20)$ per scan $(\dot{c}=300, \dot{p}=1)$.

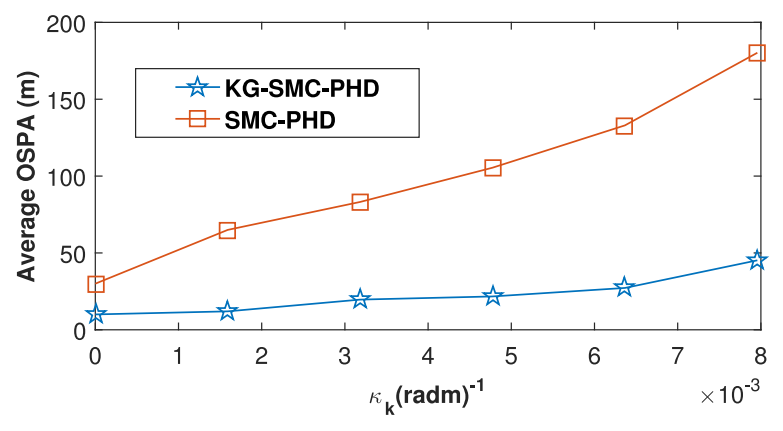

Fig. 7. Averaged OSPA distances versus varying clutter intensity over $1000 \mathrm{MC}$ trials.

correction technique. Also, in Table V, it can be observed that with just 500 particles per existing track, the KG-SMCPHD filter outperformed all other filters by having lower OSPA distance.

Fig. 6 plots the time-averaged OSPA distances for all the five filters over $1000 \mathrm{MC}$ trials with measurement set partition. Here, 1000 particles were used per existing track for the KG-SMC-PHD filter while the parameters of the other four filters were maintained. As shown in Fig. 6, high values of OSPA distance occurs when new targets are born around time indices $k=10,20,40$, and 60. It is observed from the figure that the SMC-PHD filter gave the least performance, while the proposed filter shows superior performance in terms of average OSPA distance per target when compared to the other filters under high clutter condition. The proposed filter achieved this good performance level due to our selective particle correction technique. Fig. 6 further suggests that for our simulation example, there is not a significant difference performance wise between the GM-PHD, GM-USMC-PHD, and the AP-PHD filters as all three filters gave similar level of performance in terms of average miss-distance per target.

\section{E. Overall Evaluation}

We now discuss the filter limitations in terms of OSPA distance and number of clutter points, number of particles and CT as well as general filter performance. Fig. 7 plots time averaged $1000 \mathrm{MC}$ trials of the OSPA distance for the SMC-PHD filter and the KG-SMC-PHD filter against clutter intensities from $\kappa_{k}=0(\mathrm{radm})^{-1}$ to 


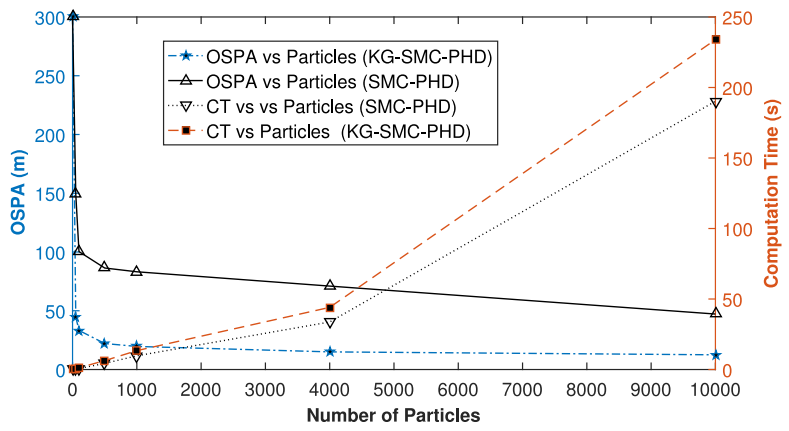

Fig. 8. KG-SMC-PHD and SMC-PHD filter performance evaluation in terms of OSPA distance and CT versus varying number of particles time averaged over $1000 \mathrm{MC}$ trials for $\lambda=20$.

$\kappa_{k}=8 \times 10^{-3}(\mathrm{radm})^{-1}$, that is, from $\lambda=0$ to $\lambda=50$. Both filters were implemented with measurement set partition with $\rho=1000$. It is observed that the miss-distance increases for both filters as clutter intensity increases. However, this increase in OSPA distance is more significant in the SMC-PHD filter implementation compared with the proposed filter. Fig. 7 clearly shows that the proposed filter outperforms the SMC-PHD filter as it maintains an average OSPA distance of less than $51 \mathrm{~m}$ up to clutter intensity of $\kappa_{k}=8 \times 10^{-3}(\mathrm{radm})^{-1}$ due to the particle state correction technique in our approach while the SMC-PHD filter starts to exhibit breakdown from about $\kappa_{k}=6.4 \times 10^{-3}(\mathrm{radm})^{-1}$ (i.e., $\lambda=40$ ). For this simulation example, the proposed filter performed well up to $\lambda=60$ and started exhibiting breakdown at about $\lambda=65$. Note that this time-averaging result is intended as a guide to provide a broad indication of the performance of the filter and can vary depending on the application scenario.

Fig. 8 shows the effect of the choice of number of particles on OSPA distance and CT. As expected, on the righthand side of the $y$-axis, the CT increases for both filters as number of particles increases. It is also observed that for the same number of particles, the CT of the SMC-PHD filter is always lower when compared to the proposed approach and the difference in $\mathrm{CT}$ for both filters increases with increase in number of particles. The extra computation load for the proposed filter is due to the extra particle state correction step of our approach. However, on the left-hand side of the $y$-axis, the miss-distance of the proposed technique is seen to be significantly lower compared to the SMC-PHD filter. Although the performance of the SMC-PHD filter is seen to improve with increase in the number of particles, the filter did not achieve the accuracy level of the proposed filter even with 10000 particles. In terms of miss-distance, Fig. 8 also suggests that the proposed filter is more efficient as only few a particles (less than 1000) are required to achieve an OSPA distance of less than $50 \mathrm{~m}$ while the SMC-PHD filter requires about 10000 particles.

Comparing the contributions of this paper, first, a partitioning technique was used on consecutive measurement sets to separate existing targets from clutter and newborn targets. This process primarily serves the function of reducing the number candidate measurements to use in the weight update stage in the presence of clutter. A reduced computational burden is thus achieved as the unnecessary computation on measurements due to clutter is avoided during weight update. Second, the Kalman gain as a correction technique seeks to achieve minimal variance and thereby gives better accuracy (in approximating the posterior). As a result, fewer particles are required to populate regions of interest. Furthermore, the effect of the partitioning process is not apparent in the no clutter to partition. However, the correction step is needed with or without clutter. As a whole, the use of the Kalman-gain correction method is the contribution which gives the main improvement.

\section{CONCLUSION}

We have proposed a new and efficient SMC-PHD filter for MTT, which seeks to minimize the MSE between received and estimated measurements at any given time. This was achieved by first partitioning the measurement set into target-originated measurements and clutter for weight computation and applying the Kalman gain to selected particles for state correction. The tracking performance was improved because only target-originated measurements were used for weight computation and the MSE at each time step was reduced resulting in fewer number of particles for state estimation. Simulation results demonstrate that our algorithm outperforms the standard SMC-PHD filter as well as other alternative implementations of the PHD filter. In our future work, the proposed filter will be extended to track maneuvering and closely spaced targets and applied to other target tracking applications including that in a multipleinput-multiple-output (MIMO) radar environment.

\section{ACKNOWLEDGMENT}

The authors would like to thank the Associate Editor and all the reviewers for their very valuable and insightful comments during the revision of this work.

\section{REFERENCES}

[1] B.-N. Vo et al. Multitarget tracking In Wiley Encyclopedia of Electrical and Electronics Engineering. Hoboken, NJ, USA: Wiley, Sep. 2015, pp. 1-15.

[2] P. N. Trung

Tracking of multiple objects using the PHD filter Ph.D. dissertation, National Univ. Singapore, Singapore, 2007.

[3] Y. Bar-Shalom, X. R. Li, and T. Kirubarajan Estimation With Applications to Tracking and Navigation: Theory Algorithms and Software. Hoboken, NJ, USA: Wiley, 2004.

[4] S. S. Blackman Multiple hypothesis tracking for multiple target tracking IEEE Aerosp. Electron. Syst. Mag., vol. 19, no. 1, pp. 5-18, Jan. 2004.

[5] Y. Bar-Shalom and X.-R. Li Multitarget-Multisensor Tracking: Principles and Techniques. Storrs, CT, USA: Univ. Connecticut Press, 1995.

[6] R. P. S. Mahler

Multitarget Bayes filtering via first-order multitarget moments IEEE Trans. Aerosp. Electron. Syst., vol. 39, no. 4, pp. 11521178, Oct. 2003. 
[7] R. P. S. Mahler

Statistics 101 for multisensor, multitarget data fusion

IEEE Aerosp. Electron. Syst. Mag., vol. 19, no. 1, pp. 53-64, Jan. 2004.

[8] R. P. S. Mahler

Statistical Multisource-Multitarget Information Fusion.

Norwood, MA, USA: Artech House, 2007.

[9] R. P. S. Mahler

"Statistics 102" for multisource-multitarget detection and track-

ing

IEEE J. Sel. Topics Signal Process., vol. 7, no. 3, pp. 376-389, Jun. 2013.

[10] R. P. S. Mahler

PHD filters of higher order in target number

IEEE Trans. Aerosp. Electron. Syst., vol. 43, no. 4, pp. 1523 1543, Oct. 2007.

[11] B.-T. Vo, B.-N. Vo, and A. Cantoni

The cardinality balanced multi-target multi-Bernoulli filter and its implementations

IEEE Trans. Signal Process., vol. 57, no. 2, pp. 409-423, Feb. 2009.

[12] B. T. Vo and B. N. Vo

Labeled random finite sets and multi-object conjugate priors

IEEE Trans. Signal Process., vol. 61, no. 13, pp. 3460-3475, Jul. 2013.

[13] B. N. Vo, B. T. Vo, and D. Phung

Labeled random finite sets and the Bayes multi-target tracking filter

IEEE Trans. Signal Process., vol. 62, no. 24, pp. 6554-6567, Dec. 2014.

[14] F. Papi, B. N. Vo, B. T. Vo, C. Fantacci, and M. Beard

Generalized labeled multi-bernoulli approximation of multiobject densities

IEEE Trans. Signal Process., vol. 63, no. 20, pp. 5487-5497, Oct. 2015.

[15] M. Beard, B. T. Vo, and B. N. Vo

Bayesian multi-target tracking with merged measurements using labelled random finite sets

IEEE Trans. Signal Process., vol. 63, no. 6, pp. 1433-1447, Mar. 2015.

[16] B.-N. Vo and W.-K. Ma

The gaussian mixture probability hypothesis density filter

IEEE Trans. Signal Process., vol. 54, no. 11, pp. 4091-4104, Nov. 2006.

[17] B.-N. Vo, S. Singh, and A. Doucet

Random finite sets and sequential Monte Carlo methods in multi-target tracking

In Proc. Int. Radar Conf., 2003, pp. 486-491.

[18] A. Doucet, S. Godsill, and C. Andrieu

On sequential Monte Carlo sampling methods for Bayesian filtering

Statist. Comput., vol. 10, no. 3, pp. 197-208, 2000.

[19] S. Maskell and S. Julier

Optimised proposals for improved propagation of multi-modal distributions in particle filters

In Proc. 16th Int. Conf. Inf. Fusion, 2013, pp. 296-303.

[20] B.-N. Vo, S. Singh, and A. Doucet

Sequential Monte Carlo methods for multitarget filtering with random finite sets

IEEE Trans. Aerosp. Electron. Syst., vol. 41, no. 4, pp. 1224 1245, Oct. 2005.

[21] J. H. Yoon, D. Y. Kim, and K.-J. Yoon

Gaussian mixture importance sampling function for unscented SMC-PHD filter

Signal Process., vol. 93, no. 9, pp. 2664-2670, 2013.

[22] N. Whiteley, S. Singh, and S. Godsill

Auxiliary particle implementation of probability hypothesis density filter

IEEE Trans. Aerosp. Electron. Syst., vol. 46, no. 3, pp. $1437-$ 1454, Jul. 2010.
[23] E. Baser and M. Efe

A novel auxiliary particle PHD filter

In Proc. 15th Int. Conf. Inf. Fusion, 2012, pp. 165-172.

[24] M. Melzi and A. Ouldali

Joint multiple target tracking and classification using the unscented Kalman particle PHD filter

in Proc. IEEE 9th Int. New Circuits Syst. Conf., 2011, pp. 534 537.

[25] W. Ma, B. Ma, and X. Zhan

Kalman particle PHD filter for multi-target visual tracking In Intelligent Science and Intelligent Data Engineering. Berlin, Germany: Springer-Verlag, 2012, pp. 341-348.

[26] X. Tang, J. Zhou, J. Huang, and P. Wei

Improved particle implementation of the probability hypothesis density filter in resampling

In Proc. IEEE 12th Int. Conf. Comput. Inf. Technol., 2012, pp. 56-61.

[27] D. Clark, B.-T. Vo, and B.-N. Vo

Gaussian particle implementations of probability hypothesis density filters

In Proc. IEEE Aerosp. Conf., 2007, pp. 1-11.

[28] J. Yin, J. Zhang, and J. Zhao

The Gaussian particle multi-target multi-bernoulli filter In Proc. 2nd Int. Conf. Adv. Comput. Control, 2010, vol. 4, pp. 556-560.

[29] J. Zhang, H. Ji, and C. Ouyang

A new Gaussian mixture particle CPHD filter for multitarget tracking

In Proc. Int. Symp. Intell. Signal Process. Commun. Syst., 2010, pp. 1-4.

[30] Y. Petetin and F. Desbouvries

A mixed GM/SMC implementation of the probability hypothesis density filter

In Proc. 11th Int. Conf. Inf. Sci., Signal Process. Appl., 2012, pp. 425-430.

[31] Z. Khan, T. Balch, and F. Dellaert

MCMC-based particle filtering for tracking a variable number of interacting targets

IEEE Trans. Pattern Anal. Mach. Intell., vol. 27, no. 11, pp. 1805-1819, Nov. 2005.

[32] Y. Zheng, Z. Shi, R. Lu, S. Hong, and X. Shen

An efficient data-driven particle PHD filter for multitarget tracking

IEEE Trans. Ind. Informat., vol. 9, no. 4, pp. 2318-2326, Nov. 2013.

[33] N. Bergman

Recursive Bayesian estimation: Navigation and tracking applications

Ph.D. dissertation, Linkoping Univ., Linkoping, Sweden, 1999.

[34] B. Ristic, S. Arulampalam, and N. Gordon

Beyond the Kalman Filter: Particle Filters for Tracking Applications. Norwood, MA, USA: Artech House, 2004.

[35] B. K. Habtemariam, R. Tharmarasa, and T. Kirubarajan PHD filter based track-before-detect for MIMO radars Signal Process., vol. 92, no. 3, pp. 667-678, 2012.

[36] S. Kay

Fundamentals of Statistical Signal Processing: Estimation Theory, vol. 1. Englewood Cliffs, NJ, USA: Prentice-Hall, 1993.

[37] D. Schuhmacher, B.-T. Vo, and B.-N. Vo

A consistent metric for performance evaluation of multi-object filters

IEEE Trans. Signal Process., vol. 56, no. 8, pp. 3447-3457, Aug. 2008. 

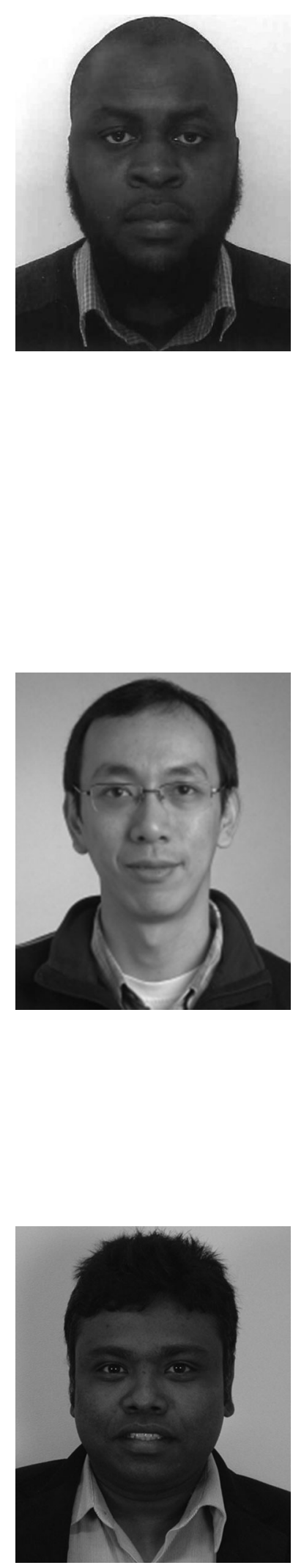

Abdullahi Daniyan (S'13) received the B.Eng. (Hons.) degree in electrical and electronic engineering from the University of Bradford, Bradford, U.K., in 2010, and the M.Sc. degree in signal processing for communication systems from Loughborough University, Loughborough, U.K., in 2014. He is currently working toward the Ph.D. degree in Signal Processing at Loughborough University.

His research interests include multiple target tracking, sensor data fusion, machine learning, linear and nonlinear filtering and estimation, and renewable energy.

Yu Gong (M'07) received the B.Eng. and M.Eng. degrees in electronic engineering from the University of Electronics and Science Technology of China, Chengdu, China, in 1992 and 1995, respectively, and the Ph.D. degree in communications from the National University of Singapore, Singapore, in 2002.

After the Ph.D. graduation, he took several research positions with the Institute of Infocomm Research, Singapore, and also with the Queen's University of Belfast, Belfast, U.K. From 2006 to 2012, he was an Academic Member with the School of Systems Engineering, University of Reading, Reading, U.K. Since July 2012, he has been with the School of Electronic, Electrical and Systems Engineering, Loughborough University, Loughborough, U.K. His research interests are in the area of signal processing and communications, including wireless communications, cooperative networks, nonlinear and nonstationary system identification, and adaptive filters.

Sangarapillai Lambotharan (SM'06) received the Ph.D. degree in signal processing from Imperial College London, London, U.K., in 1997.

He was a Postdoctoral Research Associate in the Imperial College London till 1999. He was a Visiting Scientist in the Engineering and Theory Center of Cornell University, NY, USA, in 1996. From 1999 to 2002, he was with the Motorola Applied Research Group, U.K., as a Research Engineer, working in many projects, including physical-link layer modeling and performance characterization of GPRS, EGPRS, and UTRAN. From 2002 to 2007, he was with the King's College London, U.K., and Cardiff University, U.K., as a Lecturer and Senior Lecturer, respectively. He is currently a Professor of digital communications and the Head of Signal Processing and Networks Research Group at Loughborough University, Loughborough, U.K. His current research interests include wireless communications, cognitive radio networks, smart grids, radars, convex optimizations, and game theory. He has published more than 175 conference and journal articles in these areas.

Dr. Lambotharan is an Associate Editor of the EURASIP Journal on Wireless Communications and Networking. 

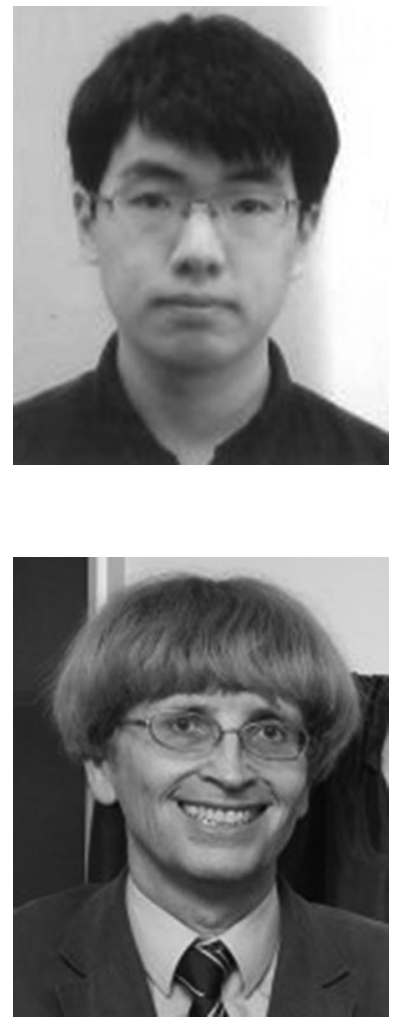

Pengming Feng (S'13-M'16) was born in Jilin, China. He received the B.Sc. degree in automatic control from the Beijing University of Chemical Technology, Beijing, China, in 2012, the M.Sc. degree in digital communication systems from Loughborough University, Loughborough, U.K., in 2013, and the Ph.D. degree in intelligent signal processing from Newcastle University, Newcastle upon Tyne, U.K., in 2016.

During the Ph.D. program, he worked as a part of the University Defence Research Collaboration, sponsored by the U.K. Defence Science and Technology Laboratory and Engineering and Physical Science Research Council, on the project entitled Signal Processing in Networked Battlespace. His research interests include multiple target tracking, machine learning, and sparse representation.

Jonathon Chambers (S'85-M'85-SM'98-F'11) received the Ph.D. and D.Sc. degrees in signal processing from the Imperial College of Science, Technology, and Medicine, Imperial College London, London, U.K., in 1990 and 2014, respectively.

From 1991 to 1994, he was a Research Scientist in the Schlumberger Cambridge Research Centre, Cambridge, U.K. In 1994, he returned to Imperial College London as a Lecturer in signal processing and was promoted to a Reader (Associate Professor) in 1998. From 2001 to 2004, he was the Director of the Centre for Digital Signal Processing and a Professor of signal processing with the Division of Engineering, Kings College London, London. From 2004 to 2007, he was a Cardiff Professorial Research Fellow in the School of Engineering, Cardiff University, Cardiff, U.K. Between 2007 and 2014, he led the Advanced Signal Processing Group, School of Electronic, Electrical, and Systems Engineering, Loughborough University, Loughborough, U.K., where he remains a Visiting Professor. In 2015, he joined the School of Electrical and Electronic Engineering, Newcastle University, Newcastle upon Tyne, U.K., where he is a Professor of signal and information processing and the Head of the Communications, Sensors, Signal and Information Processing Group. He is also a Guest Professor in Harbin Engineering University, Harbin, China. He has advised approaching 80 researchers to Ph.D. degree, and authored or coauthored more than 500 conference and journal articles, many of which are in IEEE journals. His research interests include adaptive signal processing and machine learning and their applications.

Dr. Chambers is a Fellow of the Royal Academy of Engineering, U.K. He is currently a Member of the IEEE Signal Processing Society Jack Kilby Medal Committee. He was the Technical Program Chair of the 15th International Conference on Digital Signal Processing and the 2009 IEEE Workshop on Statistical Signal Processing, both held in Cardiff, and a Technical Program Co-Chair for the 36th IEEE International Conference on Acoustics, Speech, and Signal Processing, Prague, Czech Republic. He has served on the IEEE Signal Processing Theory and Methods Technical Committee for six years, and the IEEE Signal Processing Society Awards and Conference Boards for three years. He has also been an Associate and Senior Editor of the IEEE TRAnSACtions on Signal Processing for three terms. He received the first QinetiQ Visiting Fellowship in 2007, for his outstanding contributions to adaptive signal processing and his contributions to QinetiQ, as a result of his successful industrial collaboration with the international defence systems company QinetiQ. 